\title{
The Impact of Ligand Field Symmetry on Molecular Qubit
}

\section{Coherence}

\author{
Nathanael P. Kazmierczak, Ruben Mirzoyan, and Ryan G. Hadt* \\ Division of Chemistry and Chemical Engineering, Arthur Amos Noyes Laboratory of Chemical Physics, \\ California Institute of Technology, Pasadena, CA 91125, United States
}

*Corresponding Author: rghadt@caltech.edu

\begin{abstract}
Developing quantum bits (qubits) exhibiting room temperature electron spin coherence is a key goal of molecular quantum information science. Here we develop a simple and powerful model for predicting relative $\mathrm{T}_{1}$ coherence times in transition metal complexes from dynamic ligand field principles. By considering the excited state origins of ground state spin-phonon coupling, we derive group theory selection rules governing which vibrational symmetries can induce decoherence. Thermal weighting of the coupling terms produces surprisingly good predictions of experimental $T_{1}$ trends as a function of temperature and explains previously confounding features in spin-lattice relaxation data. We use this model to evaluate experimental relaxation rates across $\mathrm{S}=1 / 2$ transition metal qubit candidates with diverse structures, gaining new insights into the interplay between spin-phonon coupling and molecular symmetry. This methodology elucidates the specific vibrational modes giving rise to decoherence, suggesting symmetry-based design
\end{abstract}


strategies and providing insight into the origin of room temperature coherence in transition metal complexes.

\section{Introduction}

The use of paramagnetic transition metal complexes as molecular electron spin quantum bits (qubits) has generated considerable interest over the past decade (Figure 1A). ${ }^{1-6}$ When placed into a magnetic field, the Zeeman effect splits the energies of the $M_{S}$ sublevels into a quantum twolevel system that can be leveraged for applications in computing, sensing, and communication (Figure 1B). ${ }^{2,7}$ Among these, molecular quantum sensing constitutes a particularly exciting application, ${ }^{2}$ as molecular electron spin qubits can be synthetically tuned and located in a targeted fashion within chemical microenvironments and interfaces to read out properties of relevance in areas such as catalysis and medicine. The microenvironments of interest often exist under ambient conditions. Thus, developing molecular qubits that operate at room temperature remains a key goal in the field. $1,8,9$

The utility of molecular electron spin qubits is limited by the phase coherence lifetime $\mathrm{T}_{2}$, which describes how long phase relations are retained between members of the ensemble. ${ }^{10}$ As temperature increases in spin-dilute environments, $\mathrm{T}_{2}$ becomes limited by $\mathrm{T}_{1}$, the spin-lattice relaxation time. $T_{1}$ describes how quickly spin energy is transferred to the vibrational bath. ${ }^{11}$ In solid lattices, this process is controlled by spin-phonon coupling. ${ }^{12}$ Three mechanisms for spinphonon coupling deteriorate the performance of molecular qubits at room temperature, known as the direct, Raman, and Orbach processes (Figure 1B). ${ }^{10,13,14}$ The direct process dissipates spin energy through acoustic phonon emission and exerts the greatest contribution at low temperatures (e.g., $<10 \mathrm{~K}) .{ }^{15}$ The Raman process dissipates spin energy through inelastic scattering of phonons 
from a virtual state, with acoustic phonons contributing at intermediate temperatures and optical phonons (i.e., local modes ${ }^{6}$ ) dominating at elevated temperatures near ambient conditions. ${ }^{12,16}$ In $\mathrm{S}=1 / 2$ systems, the Orbach mechanism generally does not contribute strongly. ${ }^{16}$ Room temperature coherence lifetimes of molecular electron spin qubits are controlled by spin-phonon coupling with the molecular vibrational modes. ${ }^{4,17}$

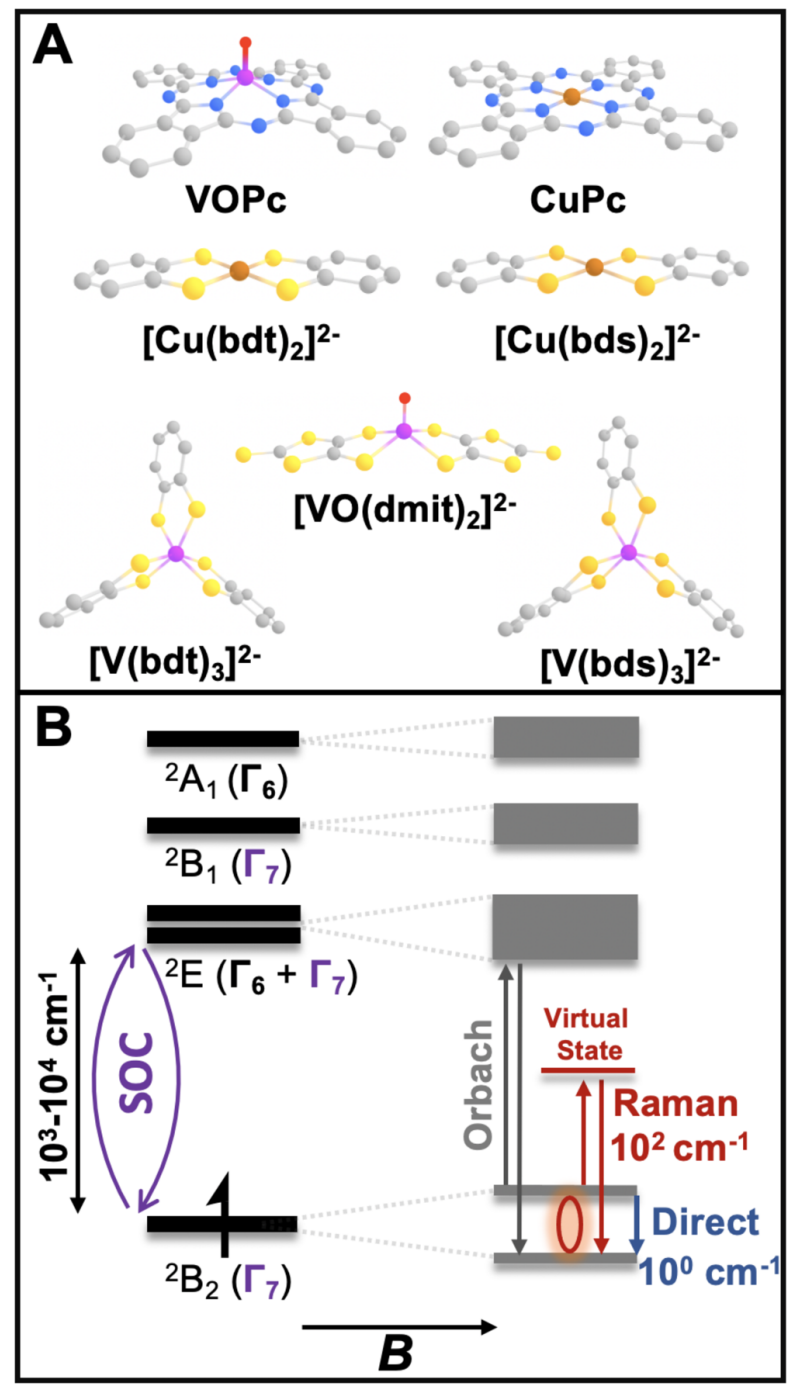

Figure 1. Overview of molecular electron spin qubits. (A) V(IV) and $\mathrm{Cu}(\mathrm{II})$ qubits considered in this study ${ }^{8,9,18,19} \mathrm{VOPc}=$ vanadyl phthalocyanine; $\mathrm{CuPc}=$ copper phthalocyanine; $\left[\mathrm{Cu}(\mathrm{bdt})_{2}\right]^{2-}=$ copper bis(1,2-benzenedithiolate); $\left[\mathrm{Cu}(\mathrm{bds})_{2}\right]^{2-}=$ copper $\quad$ bis $(1,2$-benzenediselenlate $)$; $\left[\mathrm{VO}(\mathrm{dmit})_{2}\right]^{2-}=$ vanadyl bis $(1,3-$ dithiole-2-thione-4,5-dithiolate $) ;\left[\mathrm{V}(\mathrm{bdt})_{3}\right]^{2-}=$ vanadium tris $(1,2-$ 
benzenedithiolate); $\left[\mathrm{V}(\mathrm{bds})_{3}\right]^{2-}=$ vanadium tris(1,2-benzenediselenate); (B) Electronic structure and relaxation mechanisms of molecular qubits. (Left) Electronic states (example: VOPc) in single-valued point groups and double groups (Bethe notation) inclusive of spin-orbit coupling. Charge-transfer states not shown. (Right) $\mathrm{T}_{1}$ relaxation mechanisms. Atomic color scheme: $\mathrm{C}$ (grey), $\mathrm{N}$ (blue), $\mathrm{O}$ (red), $\mathrm{S}$ (yellow), Se (orange), $\mathrm{Cu}$ (brown), $\mathrm{V}$ (pink). $\mathrm{H}$ atoms not shown for clarity.

A natural question arises: which vibrational modes exhibit the strongest spin-phonon coupling? Because the Raman process requires thermal population of an existing phonon mode, ${ }^{10}$ vibrational modes higher in frequency than $400 \mathrm{~cm}^{-1}$ are not expected to contribute significantly to the spin-lattice relaxation. While the phonon density of states and dispersion relation below 400 $\mathrm{cm}^{-1}$ can be probed using terahertz spectroscopy ${ }^{20}$ and four-dimensional inelastic neutron scattering, ${ }^{21}$ ascertaining the spin-phonon coupling of those modes remains an outstanding experimental challenge. In lieu of experimental evidence, several studies have sought to assign the most impactful spin-phonon coupling modes through computational studies. ${ }^{17,22-24}$ There exists an emerging recognition of the importance of the symmetry of the vibrational mode, with recent studies empirically concluding that gerade modes exhibit heightened spin-phonon coupling over ungerade modes for square planar compounds. ${ }^{22,24}$ However, no general theory yet exists for predicting which vibrational symmetries exert the greatest spin-phonon coupling and modeling the implications for temperature-dependent $\mathrm{T}_{1}$. This hinders rational molecular design and constitutes an important challenge in the field. ${ }^{6}$

Here we derive group theory selection rules for determining vibrational modes that are active for spin-phonon coupling. We show that the coupling modes are those that are group 
theoretically allowed to undergo ligand field excited state distortions. These vibrational modes dynamically change the amount of ground state orbital angular momentum. We then show that a simple thermal weighting of these molecular vibrational mode spin-phonon coupling coefficients furnishes an excellent agreement with experimental spin-lattice relaxation rate trends, thus describing how different vibrations dominate $\mathrm{T}_{1}$ over different temperature regimes.

\section{Results and Analysis}

2.1. Symmetry effects on spin-phonon coupling. Spin-phonon coupling arises when some portion of the spin Hamiltonian is modulated by a vibrational mode. ${ }^{6,25}$ The $g$ tensor, $\boldsymbol{g}$, describing the Zeeman effect has been implicated as a major source of spin-phonon coupling in molecular qubits. ${ }^{15,24}$ Therefore, to understand the impact of symmetry on spin-phonon coupling, we first turn to the molecular origins of the $g$ values in a transition metal complex.

A free electron has an isotropic $g$ value of $g_{e}=2.0023$ owing to its intrinsic spin angular momentum; deviations from this value arise when the electron additionally possesses ground state orbital angular momentum, as quantified by the Landé formula. While the presence of a ligand field quenches orbital angular momentum in tetragonal transition metal complexes, spin-orbit coupling with ligand field excited states reintroduces orbital angular momentum into the ground state. Thus, changes in the $g$ value arise from changes in spin-orbit coupling. In order for the $i_{t h}$ vibrational mode to have a nonzero first-order spin-phonon coupling coefficient, $\partial \boldsymbol{g} / \partial Q_{i}$, the magnitude of spin-orbit coupling must therefore change as a function of the vibrational mode coordinate $Q_{i}$. The expression for the $g$ value of a transition metal complex due to the spin-orbit perturbation is given by ${ }^{26}$ 


$$
g_{i}=g_{e}-2 \lambda \sum_{e \neq g} \frac{\left\langle\Psi_{g}\left|\hat{\boldsymbol{L}}_{\boldsymbol{i}}\right| \Psi_{e}\right\rangle\left\langle\Psi_{e}\left|\hat{\boldsymbol{L}}_{\boldsymbol{i}}\right| \Psi_{g}\right\rangle}{E_{e}-E_{g}}
$$

where $\lambda$ is the many-electron spin-orbit coupling constant, $\Psi_{g}$ and $\Psi_{e}$ are the ground and excited states with energies $E_{g}$ and $E_{e}$, respectively, $\hat{\boldsymbol{L}}_{\boldsymbol{i}}$ is an orbital angular momentum operator, and $i=$ $x, y, z$ refer to the $g$ tensor principal axes and the molecular quantization frame, which are aligned for the tetragonal qubits considered in this work. Equation 1 shows that the $g$ values have a sensitive dependence on the energy gap between the ground and excited states involved in the spin-orbit coupling. (The precise excited states involved can be determined from double groups (Figure 1B) using Tables S11 and S12 and tables of d-orbital rotations. ${ }^{6,27}$ ) If the ground and excited state potential energy surfaces reach a minimum at the same value of the vibrational coordinate $Q_{i}$, then the energy gap $E_{e}-E_{g}$ can at most vary quadratically as a function of $Q_{i}$, implying $\partial \boldsymbol{g} / \partial Q_{i}=0$ (Figure 2B). However, if the equilibrium geometry of the excited state is different than that of the ground state equilibrium geometry along $Q_{i}$, the energy gap $E_{e}-E_{g}$ can vary linearly as a function of $Q_{i}$ and give rise to $\partial \boldsymbol{g} / \partial Q_{i} \neq 0$ (Figure 2B). We refer to such modes as the distorting modes. ${ }^{27}$ The first-order coupling coefficient $\partial \boldsymbol{g} / \partial Q_{i}$ is predicted to exert the leading influence on spin-lattice relaxation times. ${ }^{15,24}$ Therefore, the most important vibrational modes for spin-phonon coupling are precisely these distorting modes. ${ }^{22}$ 
A
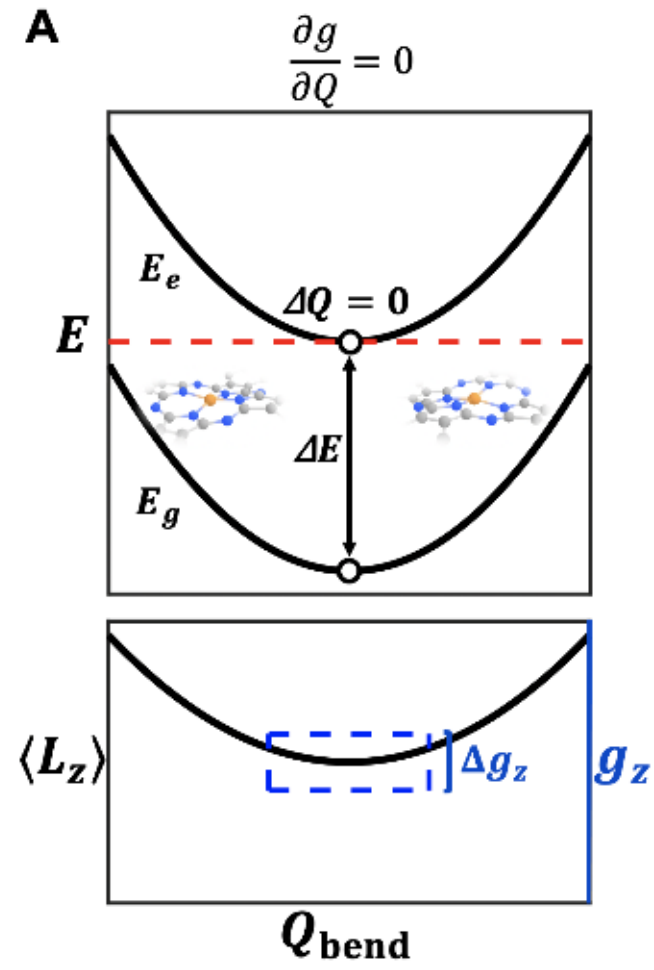

B
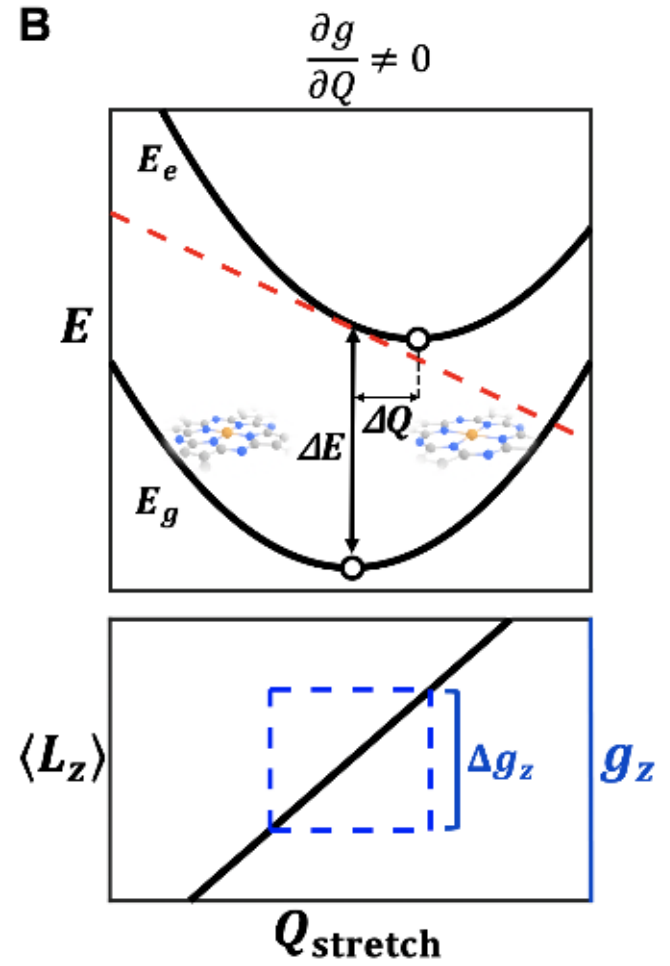

Figure 2. The excited state origins of ground state spin-phonon coupling. (A) Schematic potential energy surfaces for the $b_{2 u}$ bending mode in $\mathrm{CuPc}$. The ground and excited state potential energy minima coincide, implying no excited state distortion and thus no linear spin-phonon coupling. (B) Schematic potential energy surfaces for the $a_{1 g}$ symmetric stretch in CuPc. The ground and excited state minima are offset, implying excited state distortion and linear ground state spinphonon coupling.

Crucially, the excited state distortion can be expressed through a matrix element ${ }^{27}$ involving vibrational perturbations of the ligand field Hamiltonian $\left(H_{L F}\right)$ :

$$
\Delta Q_{i}=-\frac{\left\langle\psi_{\text {elec }}^{e}\left|\left(\frac{\partial H_{L F}}{\partial Q_{i}}\right)_{0}\right| \psi_{\text {elec }}^{e}\right\rangle}{k_{i}}
$$


Here $\Delta Q_{i}$ gives the excited state distortion along the vibrational mode $Q_{i}, k_{i}$ is the force constant, and $\psi_{\text {elec }}^{e}$ is the excited state wave function that spin-orbit couples into the ground state. The matrix element is evaluated at the ground state equilibrium geometry. The key utility of this expression lies in the application of group theory symmetry selection rules to the integral. The state symmetry of $\psi_{\text {elec }}^{e}$ and $Q_{i}\left(\Gamma_{\text {elec }}\right.$ and $\Gamma_{Q_{i}}$, respectively) can be assigned through textbook techniques. ${ }^{28}$ The ligand field Hamiltonian always has the totally symmetric irreducible representation in the molecular point group, so the derivative has the symmetry $\Gamma_{Q_{i}}$. Therefore, the symmetry of the integrand is given ${ }^{27}$ by a direct triple product. For the integral to be nonzero, Equation 3 a must contain the totally symmetric irreducible representation:

$$
\left(\Gamma_{\text {elec }} \times \Gamma_{Q_{i}} \times \Gamma_{\text {elec }}\right)=a_{1}+\cdots
$$

Here $a_{1}$ in Equation 3a signifies the totally symmetric representation in the desired point group, and the excited state is group theoretically allowed to undergo distortion when the condition in Equation $3 b$ is met:

$$
\left[\Gamma_{\text {elec }} \times \Gamma_{\text {elec }}\right]=\Gamma_{Q_{i}}
$$

The square brackets in Equation $3 \mathrm{~b}$ denote the symmetric direct product operation, appropriate for the product of $\Gamma_{\text {elec }}$ with itself, and $\Gamma_{Q_{i}}$ represents all mode symmetries that are allowed to couple. ${ }^{27,29,30}$ This selection rule enables facile calculation of which vibrational symmetries will be able to exhibit linear spin-phonon coupling terms for a given coordination geometry and 
electronic structure. This analysis shows that the critical modes to consider are those that are group theoretically allowed to undergo ligand field excited state distortions. For nondegenerate states, only the totally symmetric modes will couple, while other nontotally symmetric modes can couple for degenerate excited states. We note that this consideration is a more general basis for understanding forces in molecules (i.e., the Hellmann-Feynman force ${ }^{31}$ ), including those of relevance for transition metal photophysics ${ }^{32,33}$ and those predicted by the Jahn-Teller theorem to give rise to the instability of orbitally degenerate states. ${ }^{29}$

To illustrate the power of this approach in understanding spin-phonon coupling contributions to decoherence in molecular qubits, we turn to a comparison between vanadyl phthalocyanine (VOPc) and copper phthalocyanine (CuPc) (Figure 1A).${ }^{19} \mathrm{VOPc}$ belongs to the non-centrosymmetric point group $C_{4 v}$, while $\mathrm{CuPc}$ belongs to the centrosymmetric point group $D_{4 h}$. The electronic ground state of VOPc has the state symbol ${ }^{2} \mathrm{~B}_{2}\left(\mathrm{~d}_{\mathrm{xy}}\right)$, which spin-orbit couples with the ${ }^{2} \mathrm{~B}_{1}\left(\mathrm{~d}_{\mathrm{x} 2-\mathrm{y} 2}\right)$ excited state to introduce orbital angular momentum into $g_{z}$. The situation is reversed in CuPc owing to the hole formalism, with a ${ }^{2} \mathrm{~B}_{1 \mathrm{~g}}\left(\mathrm{~d}_{\mathrm{x} 2-\mathrm{y} 2}\right)$ ground state and a ${ }^{2} \mathrm{~B}_{2 \mathrm{~g}}\left(\mathrm{~d}_{\mathrm{xy}}\right)$ excited state. The relevant lowest lying excited state for $g_{z}$ is nondegenerate in both cases. Because the direct product of any nondegenerate irreducible representation with itself gives the totally symmetric irreducible representation, Equation $3 \mathrm{~b}$ reduces to $a_{1}=\Gamma_{Q_{i}}$ for VOPc in order for $\partial g_{z} / \partial Q_{i} \neq 0$. An identical analysis holds for $\mathrm{CuPc}$, where $a_{1 g}$ is the totally symmetric representation in $D_{4 h}$. Thus, the group theory model predicts that the strongest spin-phonon coupling for $g_{z}$ should arise from totally symmetric vibrational modes. Indeed, previous computational studies have observed that $a_{1 g}$ or $a_{1}$ modes exhibit large coupling coefficients, ${ }^{22,24}$ with $D_{2 d} \mathrm{CuCl}_{4}{ }^{2-}$ possessing more spin-phonon coupling than $D_{4 h} \mathrm{CuCl}_{4}{ }^{2-}$ owing to a greater number of totally symmetric modes. ${ }^{22}$ 
Though totally symmetric vibrational modes dominate $g_{z}$ coupling for both VOPc and $\mathrm{CuPc}$, the change in point group between $C_{4 v}$ and $D_{4 h}$ nonetheless has important consequences for spin-phonon coupling. CuPc displays a single $a_{1 g}$ mode below $400 \mathrm{~cm}^{-1}$ corresponding to the totally symmetric $\mathrm{Cu}-\mathrm{N}$ stretch (Figure 3A). Owing to the reduced number of irreducible representations in the $\mathrm{C}_{4 \mathrm{v}}$ point group, VOPc displays five total $a_{1}$ vibrational modes below 400 $\mathrm{cm}^{-1}$, encompassing mixtures of both the symmetric stretch and metal out-of-plane motion (Figure 3B). The portion of the vibrational density of states which matters for spin-phonon coupling is thus very different: CuPc possesses a lone linear coupling mode at $262 \mathrm{~cm}^{-1}$, while VOPc possesses five spin-phonon active modes below $400 \mathrm{~cm}^{-1}$ (Table 1). Calculation of the $\partial g_{z} / \partial Q_{i}$ coefficients for $\mathrm{CuPc}$ and $\mathrm{VOPc}$ via calibrated density functional theory (DFT) ${ }^{34}$ according to a previous procedure $^{22}$ (see also Supporting Information, Section 1) shows that the totally symmetric vibrations have the largest coefficients by orders of magnitude, confirming the group theory analysis (Figure 3C). The coefficient for $\mathrm{CuPc}$ is an order of magnitude larger than those for VOPc owing to the larger spin-orbit coupling constant of $\mathrm{Cu}$ (II) relative to V(IV).${ }^{19}$ For both VOPc and $\mathrm{CuPc}$, only a very small portion of the vibrational density of states contributes to spin-phonon coupling for $g_{z}($ Figure 3A,B) 


\begin{tabular}{|c|c|c|c|}
\hline \multicolumn{4}{|c|}{$\begin{array}{l}\text { Table 1. Linear } g_{z} \text { spin-phonon coupl } \\
\text { modes for VOPc and CuPc. All mode } \\
\text { totally symmetric representation. }\end{array}$} \\
\hline \multicolumn{2}{|c|}{ VOPc } & \multicolumn{2}{|c|}{$\mathrm{CuPc}$} \\
\hline $\mathrm{E}\left(\mathrm{cm}^{-1}\right)$ & $\left(\partial g_{z} / \partial Q\right)^{2}$ & $\mathrm{E}\left(\mathrm{cm}^{-1}\right)$ & $\left(\partial g_{z} / \partial Q\right)^{2}$ \\
\hline 42 & $5.5 \times 10^{-8}$ & 262 & $2.8 \times 10^{-5}$ \\
\hline 178 & $1.5 \times 10^{-6}$ & & \\
\hline 262 & $6.3 \times 10^{-7}$ & & \\
\hline 317 & $2.9 \times 10^{-6}$ & & \\
\hline 395 & $1.9 \times 10^{-6}$ & & \\
\hline
\end{tabular}




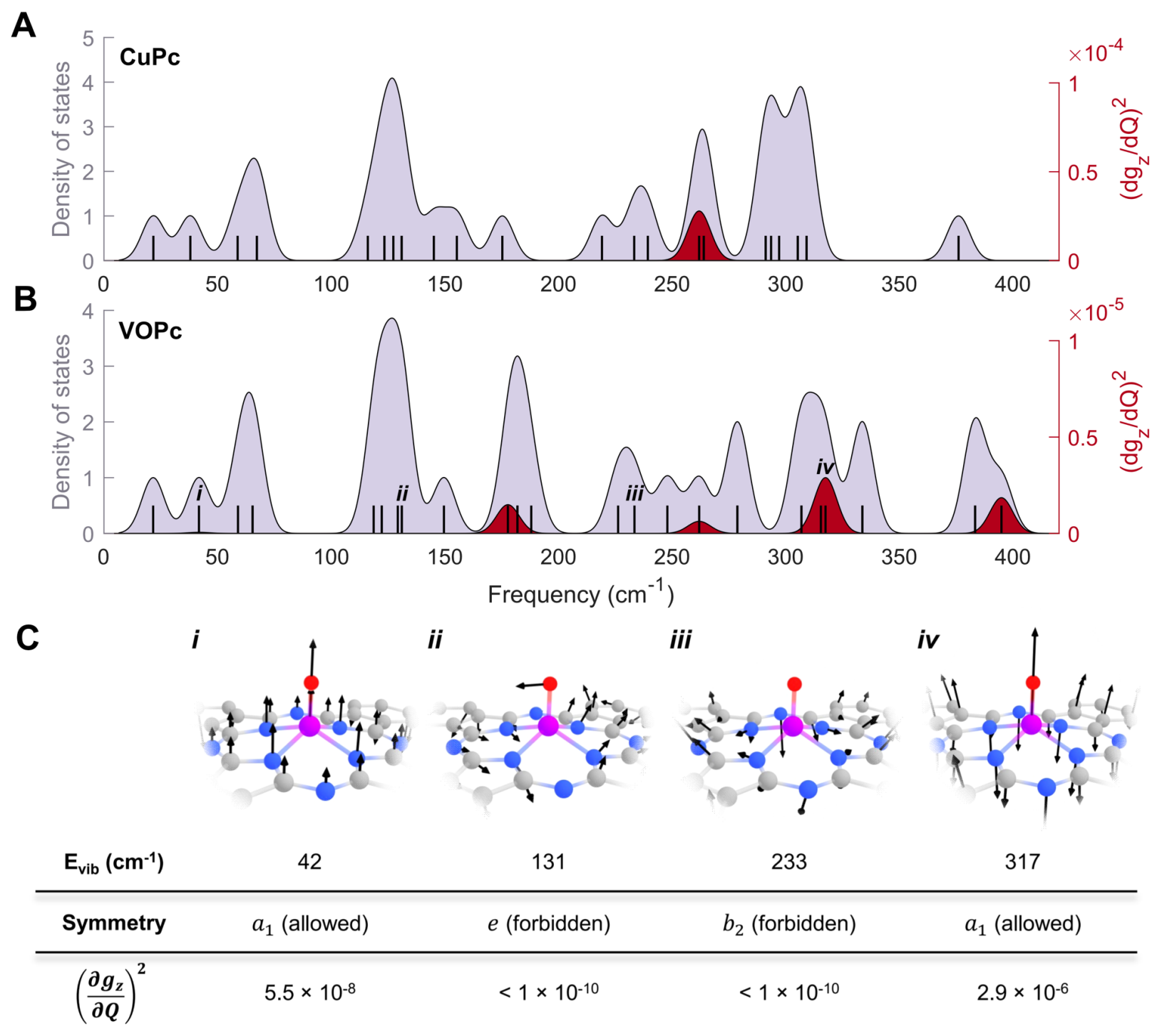

Figure 3. Impact of symmetry on spin-phonon coupling. (A) Normalized vibrational density of states (lavender, left y-axis) and spin-phonon coupling active vibrations (red, right y-axis) for CuPc. (B) Normalized vibrational density of states (lavender, left y-axis) and spin-phonon coupling active vibrations (red, right y-axis) for VOPc. (C) Analysis of selected modes for VOPc. Arrows indicate atomic displacements; additional pictures are provided in Tables S2-S6. Symmetry selection rules are evaluated for the ${ }^{2} \mathrm{~B}_{1}\left(\mathrm{~d}_{\mathrm{x} 2-\mathrm{y} 2}\right)$ excited state $\left(g_{z}\right.$ spin-phonon coupling) via Equation 3 b. $1 \times 10^{-10}$ constitutes the limit of numerical precision. 
A similar analysis can be performed for $\partial g_{x} / \partial Q_{i}$. For both VOPc and CuPc, orbital angular momentum is introduced to $g_{x}$ principally via spin-orbit coupling with the $\mathrm{d}_{\mathrm{xz}} / \mathrm{d}_{\mathrm{yz}}$ excited states, which are orbitally doubly degenerate and have the representations ${ }^{2} \mathrm{E}$ in $C_{4 v}$ and ${ }^{2} \mathrm{E}_{\mathrm{g}}$ in $D_{4 h}$. Evaluation of Equation $3 \mathrm{~b}$ for VOPc now yields $\left(a_{1}+b_{1}+b_{2}\right)=\Gamma_{Q_{i}}$, showing that $a_{1}, b_{1}$, and $b_{2}$ vibrational modes are able to have $\partial g_{x} / \partial Q_{i} \neq 0$ by symmetry. ( $a_{2}$ is produced by the antisymmetric direct product and is therefore discarded.) ${ }^{30}$ Similarly, Equation $3 \mathrm{~b}$ for CuPc yields $\left(a_{1 g}+b_{1 g}+b_{2 g}\right)=\Gamma_{Q_{i}}$, showing that multiple nondegenerate gerade modes are able to couple for $g_{x}$. Note that the gerade selection rule would hold true even if the electronic state symmetry were ungerade, because Equation $3 \mathrm{~b}$ contains the electronic symmetry twice. While group theory states which modes are allowed to couple by symmetry, as with any selection rule, this does not guarantee a large nonzero coefficient. ${ }^{27}$

Comparison between the coupling modes for $\mathrm{CuPc}$ and $\left[\mathrm{Cu}(\mathrm{bdt})_{2}\right]^{2-}(\mathrm{bdt}=1,2-$ benzenedithiolate) illustrates the impact of descending in symmetry from $D_{4 h}$ to $D_{2 h}$ (Figure 4). Lower than $400 \mathrm{~cm}^{-1}, \mathrm{CuPc}$ displays a single active mode with $\partial g_{z} / \partial Q_{i}$, the $a_{1 g}$ symmetric stretch. Two modes for CuPc display nonzero $\partial g_{x} / \partial Q_{i}$, including both the $a_{1 g}$ symmetric stretch and the $b_{1 g}$ antisymmetric stretching mode. The presence of the linearly coupling $b_{1 g}$ mode is enabled by the degeneracy of the ${ }^{2} \mathrm{E}_{\mathrm{g}}$ electronic state. However, no degenerate irreducible representations exist in the $D_{2 h}$ point group, so the $\mathrm{d}_{\mathrm{xz}}$ and $\mathrm{d}_{\mathrm{yz}}$ orbitals are split into the $\mathrm{B}_{2 g}$ and $\mathrm{B}_{3 g}$ representations. All electronic states implicated in the $g_{x}$ and $g_{y}$ spin-phonon coupling are nondegenerate for $\left[\mathrm{Cu}(\mathrm{bdt})_{2}\right]^{2-}$, implying that only totally symmetric $a_{g}$ vibrational modes will display linear coupling for all three canonical orientations. Indeed, examination of the spin-phonon coupling coefficients for $\left[\mathrm{Cu}(\mathrm{bdt})_{2}\right]^{2-}$ shows that the most prominent coupling modes are the same 
for both $\partial g_{z} / \partial Q_{i}$ and $\partial g_{x} / \partial Q_{i}$ and possess $a_{g}$ symmetry as predicted (Figure 4). The coupling $b_{1 g}$ mode from $\mathrm{CuPc}$ correlates to a $b_{1 \mathrm{~g}}$ mode in $\left[\mathrm{Cu}(\mathrm{bdt})_{2}\right]^{2-}$, implying that the linear coupling of this antisymmetric stretch mode has been turned off by the descent in symmetry. Conversely, the $b_{2 g}$ in-plane scissoring mode in $\mathrm{CuPc}$ correlates to $a_{g}$ symmetry for $\left[\mathrm{Cu}(\mathrm{bdt})_{2}\right]^{2-}$ and is activated for $g_{z}$ coupling. Thus, descent in symmetry from $D_{4 h}$ to $D_{2 h}$ retains the total number of linear coupling modes for $g_{x}$, but changes the identity of those modes (Figure 4). Similar behavior is observed for the $C_{2 v}$ qubit [ $\left.\mathrm{VO}(\mathrm{dmit})_{2}\right]^{2-}$, with many $a_{1}$ modes exhibiting coupling for both $g_{x}$ and $g_{z}$. Global molecular symmetry can impact the spin-phonon coupling modes even for apparently similar coordination geometries, a surprising result elucidated by group theory. This result establishes control of degenerate electronic excited states as an important design consideration for controlling activation of spin-phonon coupling vibrational modes.

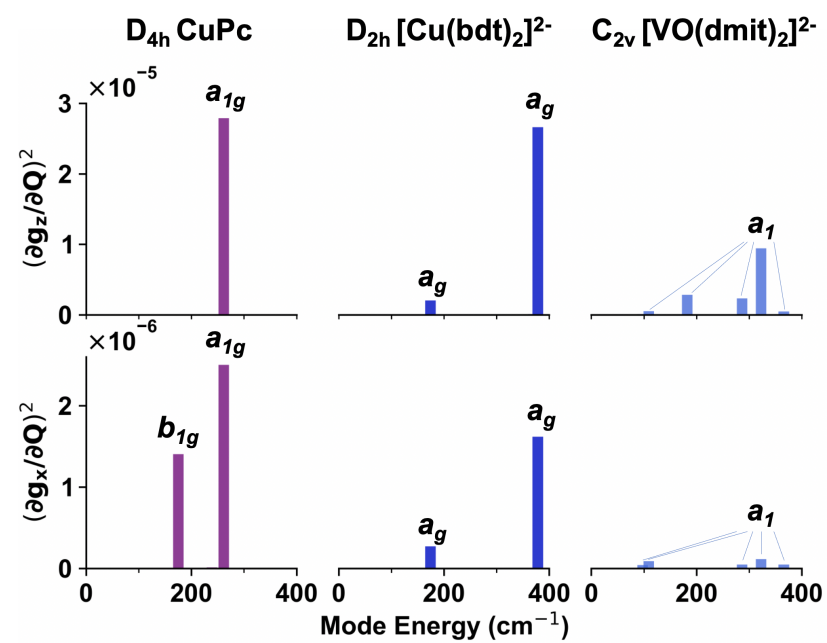

Figure 4. Orientation-dependent spin-phonon coupling coefficients for $\mathrm{CuPc},\left[\mathrm{Cu}(\mathrm{bdt})_{2}\right]^{2-}$, and $\left[\mathrm{VO}(\mathrm{dmit})_{2}\right]^{2-}$. 
A previous study of two $D_{4 h} \mathrm{Cu}(\mathrm{II})$ complexes empirically concluded that gerade modes exhibited the strongest coupling. ${ }^{24}$ Our work differs in two important ways. First, the present approach provides a predictive group theory analysis not dependent on a centrosymmetric point group. In addition to the $C_{n v}$ point groups consider in this work, this will also enable extension of spin-phonon coupling symmetry analysis to qubits with trigonal coordination environments. . $^{35,36}$ By analogy to gerade/ungerade, point groups containing the prime/double prime representations should see coupling only from the single-prime vibrational modes, as the double direct product of the electronic excited state in Equation $3 \mathrm{~b}$ will yield a single-prime representation irrespective of the electronic representation, and the totally symmetric representation will always have a singleprime value. Furthermore, evaluation of Equation $3 \mathrm{~b}$ for the $D_{4 h}$ point group reveals that the $a_{2 g}$ mode is not predicted to exhibit linear coupling despite possessing gerade symmetry. This prediction is in agreement both with previous calculations ${ }^{24}$ and our own.

Second, a point of variance with the previous study ${ }^{24}$ arises over the role of the degenerate $e_{g}$ vibrations, which are found to couple in that study, but not predicted to couple by the present group theory analysis. This is because the present analysis has considered the spin-phonon coupling coefficients corresponding to the canonical orientations of the $g$ tensor; namely, $g_{x}, g_{y}$, and $g_{z}$. By contrast, Santanni et al. averaged all nine $\partial \boldsymbol{g} / \partial Q$ values for the non-diagonalized $g$ tensor. ${ }^{24}$ Nonzero off-diagonal derivatives correspond to dynamic rotation of the principal axes of the $g$ tensor. Indeed, the $R_{x}$ and $R_{y}$ rotation operators transform as $E_{g}$ in $D_{4 h}$, and pictures of the $e_{g}$ vibrational modes show that the first coordination sphere undergoes a rigid rotation out of the xy-plane (Table S4). A minimal square-planar coordination environment such as $D_{4 h} \mathrm{CuCl}_{4}{ }^{2-}$ does not posses $e_{g}$ normal modes, ${ }^{22}$ as these would correspond to pure rotational degrees of freedom. In $\mathrm{CuPc}$, however, counterrotation of the phthalocyanine ligand framework enables $e_{g}$ normal 
modes. As local rotation does not affect bonding in the first coordination sphere, $e_{g}$ vibrational modes do not dynamically alter $g_{x}, g_{y}$, and $g_{z}$, in accordance with our group theory predictions. Similarly, the non-coupling $a_{2 g}$ modes transform as $R_{z}$.

The choice of which $\partial \boldsymbol{g} / \partial Q$ values to include in a spin relaxation model is rendered unclear by the construction of the spin Hamiltonian. ${ }^{37}$ Off-diagonal derivatives alone might be predicted to couple by Fermi's golden rule or spin-boson models, ${ }^{38}$ as these lead to matrix elements connecting $M_{S}$ states in the Zeeman term of the spin Hamiltonian. However, variation of spin-orbit coupling along the vibrational coordinate must necessarily mix the spin eigenstate composition through vector coupling of angular momenta, enabling on-diagonal derivatives such as $\partial g_{z} / \partial Q$ to induce spin relaxation. We take the view that the on-diagonal elements dominate the relaxation behavior observed for $\mathrm{S}=1 / 2$ systems, owing to (i) the known correlation between $\mathrm{T}_{1}$ times and static (i.e., canonical orientation) $\mathrm{g}$ values, ${ }^{16,23,39}$ and (ii) the superior fit to experimental temperature-dependent $\mathrm{T}_{1}$ times by models including only the on-diagonal elements (vide infra). Within the confines of the canonical orientation $\partial \boldsymbol{g} / \partial Q$ values, the present group theory approach provides the procedure for determining the modes with first-order spin-phonon coupling coefficients.

2.2. Thermally-weighted ligand field model of $\mathbf{T}_{\mathbf{1}}$. Once the $\partial \boldsymbol{g} / \partial Q$ values for molecular vibrations have been calculated, ${ }^{22}$ relative $\mathrm{T}_{1}$ times can be predicted using a simplified model of the Raman spin-lattice relaxation process in molecular solids. A simple functional form for attributing Raman relaxation to molecular vibrations has been proposed on the basis of the twophonon Green's function ${ }^{12}$ and used to fit experimental $\mathrm{T}_{1}$ data. ${ }^{24,40}$ We now employ this form to make comparative $T_{1}$ predictions informed by the preceding symmetry analysis: 


$$
\frac{1}{T_{1}} \propto \sum_{i=1}^{3 N-6}\left(\frac{\partial \boldsymbol{g}}{\partial Q_{i}}\right)^{2} \frac{\exp \left[E_{i} / k_{B} T\right]}{\left(\exp \left[E_{i} / k_{B} T\right]-1\right)^{2}}
$$

Here $E_{i}$ is the energy of the lattice vibration, $k_{B}$ is the Boltzmann constant, $T$ is the lattice temperature, and the sum is over all normal modes of vibration. Modes without a linear coupling term do not contribute to the sum. Owing to the exponentially vanishing thermal weighting factor, it is sufficient to consider only modes below $400 \mathrm{~cm}^{-1}$. Here we present rate predictions using $\partial g_{z} / \partial Q_{i}$, while predictions using other elements of the Zeeman tensor are discussed in the Supporting Information Section 3 (Figures S9-S15). Equations 3 and 4 together provide an analytical link between molecular vibrations and temperature dependent electron spin relaxation rates.
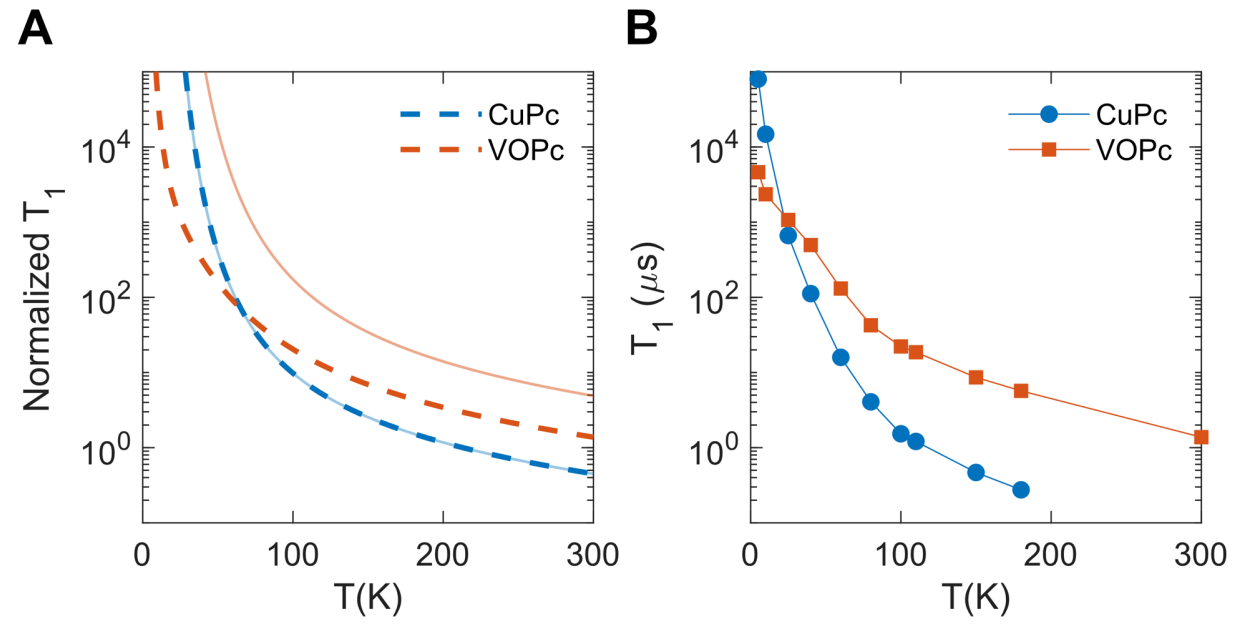

Figure 5. Thermally-weighted ligand field model for phthalocyanine qubits. (A) $\mathrm{T}_{1}$ predictions. Dashed lines: all spin-phonon active modes. Solid lines: only the two strongest modes at $317 \mathrm{~cm}^{-}$ ${ }^{1}$ and $395 \mathrm{~cm}^{-1}$ for VOPc and the single strongest mode at $262 \mathrm{~cm}^{-1}$ for CuPc . All $\mathrm{T}_{1}$ predictions 
are normalized by the same factor, chosen for the VOPc all-modes prediction to match the experimental data at $300 \mathrm{~K}$. (B) Comparison to our previous experimental results from ref ${ }^{19}$.

Figure 5A shows the predicted temperature-dependent $\mathrm{T}_{1}$ times for VOPc and $\mathrm{CuPc}$, which are in excellent agreement with our previously obtained experimental data ${ }^{19}$ (Figure 5B) considering the simplicity of the model employed. Equation 4 correctly predicts that VOPc has a longer $\mathrm{T}_{1}$ than $\mathrm{CuPc}$ at room temperature. Furthermore, Equation 4 correctly predicts the existence of a $\mathrm{T}_{1}$ crossover point at lower temperatures, below which CuPc displays the longer $\mathrm{T}_{1}$ time. Though observed in multiple systems in the molecular qubit literature, ${ }^{9,19}$ such crossover features have lacked a clear interpretation and have been attributed to variations in the Raman exponent under a Debye model treatment or local mode terms. ${ }^{16,41,42}$

We now show this phenomenon has a direct chemical interpretation in terms of molecular vibrations. As given in Table 1, VOPc possesses five linear coupling modes, while CuPc possesses only one. However, the magnitude of the spin-phonon coupling coefficient is significantly larger for the $\mathrm{CuPc}$ mode than for any of the VOPc modes, a fact explained by the difference in spinorbit coupling coefficients between the two metals. ${ }^{19,22}$ Additionally, the lone CuPc mode sits higher in energy than three of the five VOPc modes. Thus, at the lowest temperatures modeled, the symmetric stretch of $\mathrm{CuPc}$ has negligible thermal population and minimal spin-phonon coupling. By contrast, VOPc possesses coupling modes as low as $42 \mathrm{~cm}^{-1}$ (Figure 3, Table 1), which are thermally populated at low temperature and contribute to VOPc having a shorter $\mathrm{T}_{1}$ than CuPc. As the temperature increases, higher energy vibrational modes of both VOPc and CuPc become thermally populated, but the spin-phonon coupling coefficient is largest for the $\mathrm{CuPc}$ symmetric stretch. This manifests in a larger $\mathrm{T}_{1}$ slope for $\mathrm{CuPc}$ versus VOPc. When all modes are 
populated near room temperature, the larger $\partial g_{z} / \partial Q_{i}$ of CuPc takes over, and VOPc has the longer coherence time at room temperature. The high and low temperature behavior of $T_{1}$ thus relate to the magnitude of $\partial g_{z} / \partial Q_{i}$ and the relative energy of the coupling vibrational modes, respectively. We note that the precise location of the crossover point likely also contains contributions from varying efficiencies of the direct process. This may relate to effective acoustic phonon symmetry in the 1:1000 magnetic dilution data modeled here, as the $42 \mathrm{~cm}^{-1}$ linear coupling mode in VOPc contains displacements similar to an acoustic phonon (Figure 3C).

The crossover behavior predicted in the model can be unambiguously assigned to the lowenergy $a_{1}$ modes of VOPc by artificially manipulating the number of modes in the model. If only the two strongest-coupling modes of VOPc are considered $\left(317 \mathrm{~cm}^{-1}\right.$ and $\left.395 \mathrm{~cm}^{-1}\right)$, no crossover is observed (solid orange line, Figure 5A). Indeed a crossover is barely observed upon simply deleting the $a_{1}$ mode at $42 \mathrm{~cm}^{-1}$, indicating that low energy molecular vibrations produced by reduced symmetry can exert a large influence on the temperature-dependent $T_{1}$ times even when their spin-phonon coupling coefficients are small. The overall good agreement lends credence to the general use of this model to a priori predict the observation of room temperature coherence in any transition metal complex. Note that when modes of $e_{g}$ and $e$ symmetry (local rotations, vide supra) are included in the model through off-diagonal $g$ tensor derivatives, they dominate the $\mathrm{T}_{1}$ behavior for $\mathrm{CuPc}$ through thermal population owing to their low vibrational energy. ${ }^{24}$ This eliminates the predicted $T_{1}$ crossover and fails to account for the power law exponents in the intermediate-temperature regime (50 - 125K; see Figures S9 - S15 and discussion), further motivating our choice to use only the canonical $g$ value derivatives.

To demonstrate the broad applicability of the thermally-weighted ligand field model, we provide $\mathrm{T}_{1}$ predictions for $\left[\mathrm{V}(\mathrm{bdt})_{3}\right]^{2-},\left[\mathrm{Cu}(\mathrm{bdt})_{2}\right]^{2-},\left[\mathrm{V}(\mathrm{bds})_{3}\right]^{2-}$, and $\left[\mathrm{Cu}(\mathrm{bds})_{2}\right]^{2-}(\mathrm{bds}=1,2-$ 
benzenediselenate). Figure 6 shows the model predicts the same order of experimental high temperature $\mathrm{T}_{1}$ times observed previously: ${ }^{9}\left[\mathrm{Cu}(\mathrm{bdt})_{2}\right]^{2-}>\left[\mathrm{Cu}(\mathrm{bds})_{2}\right]^{2-}>\left[\mathrm{V}(\mathrm{bdt})_{3}\right]^{2-}>\left[\mathrm{V}(\mathrm{bds})_{3}\right]^{2-}$ Interestingly, the model predicts a near $\mathrm{T}_{1}$ crossover between $\left[\mathrm{Cu}(\mathrm{bds})_{2}\right]^{2-}$ and $\left[\mathrm{V}(\mathrm{bdt})_{3}\right]^{2-}$ around $100 \mathrm{~K}$, as observed experimentally at $60 \mathrm{~K}$. In the high temperature regime, $\left[\mathrm{Cu}(\mathrm{bds})_{2}\right]^{2-}$ is predicted to have a shallower slope than both $\left[\mathrm{V}(\mathrm{bdt})_{3}\right]^{2-}$ and $\left[\mathrm{Cu}(\mathrm{bdt})_{2}\right]^{2-}$, but a lower intercept than $\left[\mathrm{Cu}(\mathrm{bdt})_{2}\right]^{2-}$. Substitution of selenium for sulfur decreases the $\partial g_{z} / \partial Q_{i}$ value for the $\left[\mathrm{Cu}(\mathrm{bds})_{2}\right]^{2-}$ symmetric stretch relative to $\left[\mathrm{Cu}(\mathrm{bdt})_{2}\right]^{2-}$, but also lowers the energy of that vibrational mode. The onset of symmetric stretch spin-phonon coupling thus occurs at lower temperature in $\left[\mathrm{Cu}(\mathrm{bds})_{2}\right]^{2-}$ than $\left[\mathrm{V}(\mathrm{bdt})_{3}\right]^{2-}$, but the high temperature magnitude of spin phonon coupling is greater in $\left[\mathrm{V}(\mathrm{bdt})_{3}\right]^{2-}$ than $\left[\mathrm{Cu}(\mathrm{bds})_{2}\right]^{2-}$ owing to the larger coefficients (Tables S7-S8), leading to the near $\mathrm{T}_{1}$ crossover.
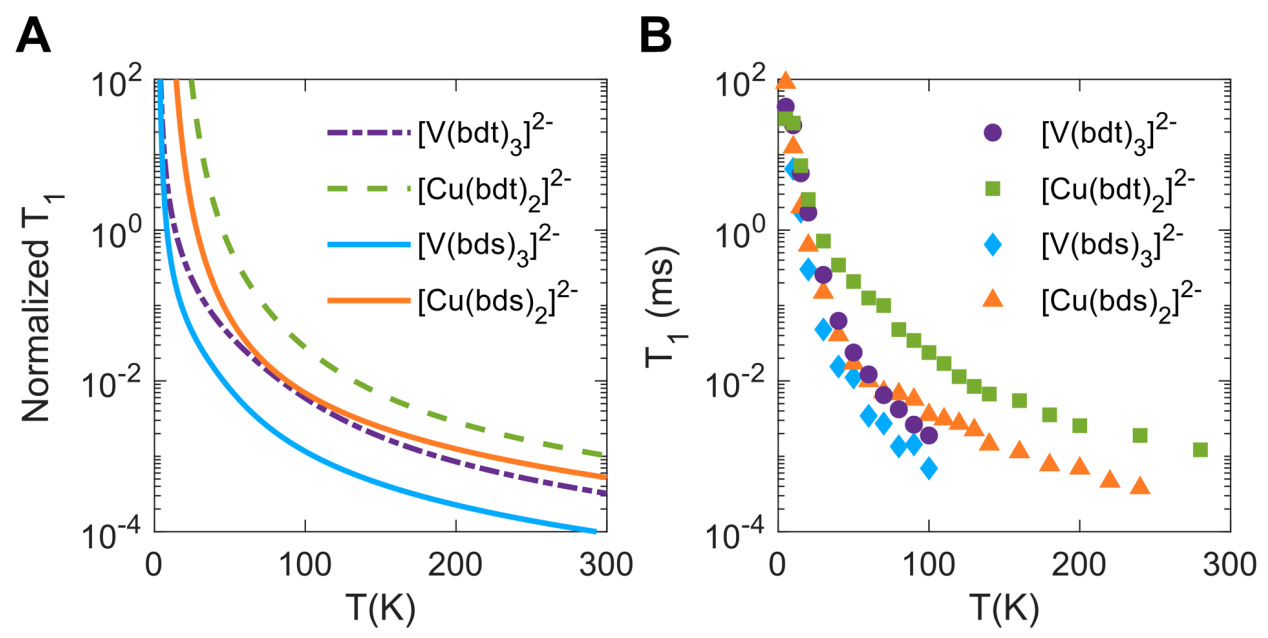

Figure 6. Thermally-weighted ligand field model for dithiolate and diselenate qubits. (A) $T_{1}$ predictions according to Equation 4. All $\mathrm{T}_{1}$ predictions are normalized by the same factor, chosen to match the experimental data for $\left[\mathrm{Cu}(\mathrm{bdt})_{2}\right]^{2-}$ at $280 \mathrm{~K}$. (B) Comparison to experimental results from ref ${ }^{9}$. 


\section{Discussion}

It has become commonplace to fit temperature-dependent spin-lattice relaxation data with a set of polynomial and exponential functions derived from the Debye model description of direct, Raman, Orbach, and local mode relaxation processes. These fits yield values such as the Debye frequency and the Raman exponent. However, recent literature has increasingly demonstrated that Debye model parameters have no unambiguous chemical interpretation for molecular solids, as the Debye model makes incompatible assumptions regarding the nature of crystalline vibrations. ${ }^{6}$ This hinders rational molecular design for quantum information science. A new molecular paradigm based on symmetry and vibrational principles is required. ${ }^{4,6,24}$

We argue the present study provides a novel and attractive perspective for modeling $\mathrm{T}_{1}$ on distinctly chemical grounds. Dynamic ligand field theory successfully predicts the magnitude ${ }^{22}$ and symmetry-based selection rules for the spin-phonon coupling coefficients. Coupled with thermal weighting, this model successfully predicts relative $T_{1}$ trends and crossovers for a variety of structurally diverse molecular qubits. The group theory selection rules and functional forms employed for temperature-dependent $T_{1}$ times are explicitly grounded in physical quantities for molecular solids, unlike in the Debye model. Previous work has considered the role of bonding descriptors such as covalency, excited state energy, and the spin-orbit coupling constant in predicting the overall magnitudes of the spin-phonon coupling coefficients between different molecules. ${ }^{19,22,23}$ These insights can be integrated with the group theory and thermal weighting approaches described herein. We anticipate that this group theory methodology will yield similar insights into the molecular origins of $\mathrm{T}_{1}$ times across a broad range of molecular electron spin qubits, including applications to $S>1 / 2$ systems. 


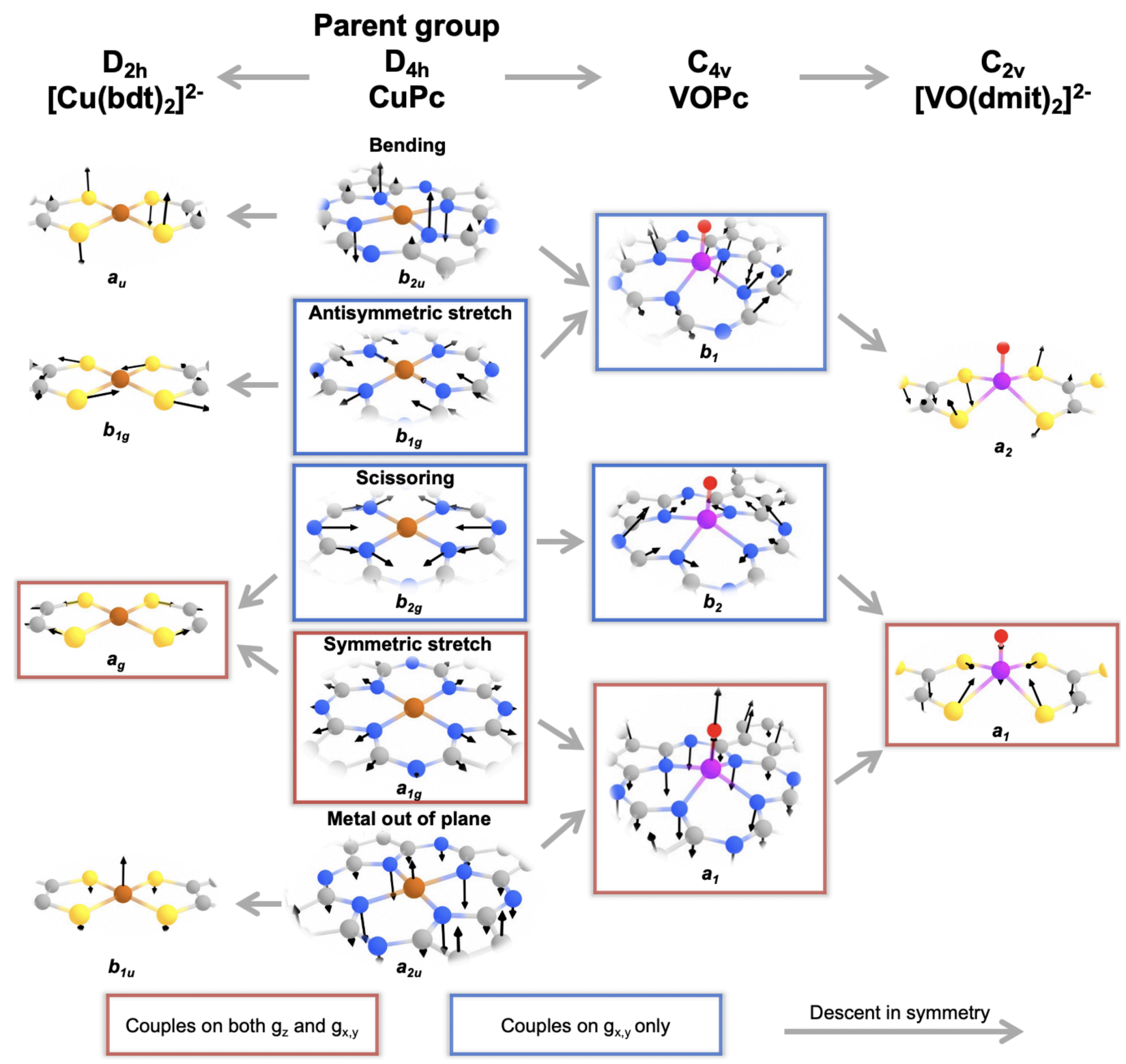

Figure 7: Symmetry flowchart of spin-phonon coupling coefficients. Convergent arrows indicate that vibrational modes mix under reduced-symmetry point groups, and boxes indicate the selection rules derived from Equation $3 b$.

In this study, we have analyzed archetypal qubits from four point groups: $D_{4 h}, D_{2 h}, C_{4 v}$, and $C_{2 v}$. Because $D_{2 h}, C_{4 v}$ and $C_{2 v}$ are all subgroups of $D_{4 h}$, the impact of symmetry on spin-phonon coupling can be broadly understood by descent in symmetry on the CuPc structure (Figure 7). 
True $D_{4 h}$ complexes such as $\mathrm{CuPc}$ and $D_{4 h} \mathrm{CuCl}_{4}{ }^{2-}$ exhibit only a single $g_{z}$-active mode in the thermally accessible region, corresponding to the $a_{1 g}$ totally symmetric ligan-metal stretch (Table S4). The $b_{1 g}$ antisymmetric ligand-metal stretch and the $b_{2 g}$ scissoring mode are also able to couple for $g_{x}$. Descent in symmetry to $C_{4 v}$ activates the $a_{2 u}\left(D_{4 h}\right)$ out-of-plane modes, which transform as $a_{1}$ in $C_{4 v}$. Phthalocyanine ligand scaffolds support many such low-energy $a_{2 u}$ modes, with CuPc possessing four $a_{2 u}$ modes below $400 \mathrm{~cm}^{-1}$ (Table S4). These are activated for coupling in VOPc (Table 1, Table S3), resulting in a smaller $\mathrm{T}_{1}$ slope than CuPc and a characteristic crossover point. Descent in symmetry to $D_{2 d}$ is known to activate new modes for spin-phonon coupling, as the $b_{2 u}$ bending mode in $D_{4 h}$ transforms as $a_{1}$ in the distorted $D_{2 d}$ point group. ${ }^{22}$ Descent in symmetry to $D_{2 h}$ shuts down $g_{x}$ spin-phonon coupling for the antisymmetric stretch $b_{1 g}$ mode while activating $g_{z}$ coupling for the $b_{2 g}\left(D_{4 h}\right)$ scissoring mode, which transforms as $a_{g}$ in $D_{2 h}$. The resulting $a_{g}$ modes contain a mixture of symmetric stretch and scissoring character. This suggests that spin-phonon coupling could be decreased by selectively hindering bond-anglealtering modes in lower symmetry point groups, a novel symmetry-based design strategy for molecular qubits.

In summary, we have developed a novel thermally-weighted dynamic ligand field model to describe and ultimately predict $T_{1}$ in molecular electron spin qubit candidates. Simplicity makes it accessible to researchers with diverse backgrounds. The methodology has allowed for the determination of the specific vibrational modes that give rise to decoherence in the $\mathrm{T}_{1}$-limited regime, ultimately elucidating the critical spin-phonon coupling, chemical bonding, and symmetry factors leading to room temperature coherence. It can be employed to a priori predict the observation of this phenomenon in any $\mathrm{S}=1 / 2$ transition metal complex. Group theory prediction of anisotropic spin-phonon coupling coefficients may prove particularly important in the context 
of quantum sensing, where anisotropic $g$ values provide a key motivation for employing transition metal complexes as versatile molecular quantum sensors.

\section{Acknowledgments}

NPK acknowledges support by the National Science Foundation Graduate Research Fellowship under Grant No. DGE-1745301. This paper's computational modeling was supported in part by National Science Foundation MRI-grant 1726260 based at Calvin University in Grand Rapids, MI, USA. Financial support from Caltech and the Dow Next Generation Educator Fund is gratefully acknowledged.

\section{References:}

(1) Atzori, M.; Sessoli, R. The Second Quantum Revolution: Role and Challenges of Molecular Chemistry. J. Am. Chem. Soc. 2019, 141 (29), 11339-11352. https://doi.org/10.1021/jacs.9b00984.

(2) Wasielewski, M. R.; Forbes, M. D. E.; Frank, N. L.; Kowalski, K.; Scholes, G. D.; YuenZhou, J.; Baldo, M. A.; Freedman, D. E.; Goldsmith, R. H.; Goodson, T.; Kirk, M. L.; McCusker, J. K.; Ogilvie, J. P.; Shultz, D. A.; Stoll, S.; Whaley, K. B. Exploiting Chemistry and Molecular Systems for Quantum Information Science. Nat. Rev. Chem. 2020, 4 (9), 490-504. https://doi.org/10.1038/s41570-020-0200-5.

(3) Graham, M. J.; Zadrozny, J. M.; Fataftah, M. S.; Freedman, D. E. Forging Solid-State Qubit Design Principles in a Molecular Furnace. Chem. Mater. 2017, 29 (5), 1885-1897. https://doi.org/10.1021/acs.chemmater.6b05433.

(4) Escalera-Moreno, L.; Baldoví, J. J.; Gaita-Ariño, A.; Coronado, E. Spin States, Vibrations and Spin Relaxation in Molecular Nanomagnets and Spin Qubits: A Critical Perspective. Chem. Sci. 2018, 9 (13), 3265-3275. https://doi.org/10.1039/C7SC05464E.

(5) Aromí, G.; Aguilà, D.; Gamez, P.; Luis, F.; Roubeau, O. Design of Magnetic Coordination Complexes for Quantum Computing. Chem. Soc. Rev. 2012, 41 (2), 537-546. https://doi.org/10.1039/C1CS15115K.

(6) Mirzoyan, R.; Kazmierczak, N. P.; Hadt, R. G. Deconvolving Contributions to Decoherence in Molecular Electron Spin Qubits: A Dynamic Ligand Field Approach. Chem. - Eur. J. https://doi.org/10.1002/chem.202100845. 
(7) Nielsen, M. A.; Chuang, I. L. Quantum Computation and Quantum Information: 10th Anniversary Edition, 1st edition.; Cambridge University Press: Cambridge ; New York, 2010.

(8) Atzori, M.; Tesi, L.; Morra, E.; Chiesa, M.; Sorace, L.; Sessoli, R. Room-Temperature Quantum Coherence and Rabi Oscillations in Vanadyl Phthalocyanine: Toward Multifunctional Molecular Spin Qubits. J. Am. Chem. Soc. 2016, 138 (7), 2154-2157. https://doi.org/10.1021/jacs.5b13408.

(9) Fataftah, M. S.; Krzyaniak, M. D.; Vlaisavljevich, B.; Wasielewski, M. R.; Zadrozny, J. M.; Freedman, D. E. Metal-Ligand Covalency Enables Room Temperature Molecular Qubit Candidates. Chem. Sci. 2019, 10 (27), 6707-6714. https://doi.org/10.1039/C9SC00074G.

(10) Standley, K. J.; Vaughan, R. A. Electron Spin Relaxation Phenomena in Solids; Springer US: Boston, MA, 1969. https://doi.org/10.1007/978-1-4899-6539-4.

(11) Bloch, F. Nuclear Induction. Phys. Rev. 1946, 70 (7-8), 460-474. https://doi.org/10.1103/PhysRev.70.460.

(12) Lunghi, A.; Sanvito, S. The Limit of Spin Lifetime in Solid-State Electronic Spins. J. Phys. Chem. Lett. 2020, 11 (15), 6273-6278. https://doi.org/10.1021/acs.jpclett.0c01681.

(13) Van Vleck, J. H. Paramagnetic Relaxation Times for Titanium and Chrome Alum. Phys. Rev. 1940, 57 (5), 426-447. https://doi.org/10.1103/PhysRev.57.426.

(14) Orbach, R. Spin-Lattice Relaxation in Rare-Earth Salts. Proc. R. Soc. Lond. A 1961, 264, 458-484.

(15) Lunghi, A.; Sanvito, S. How Do Phonons Relax Molecular Spins? Sci. Adv. 2019, 5 (9), eaax7163. https://doi.org/10.1126/sciadv.aax7163.

(16) Zhou, Y.; Bowler, B. E.; Eaton, G. R.; Eaton, S. S. Electron Spin Lattice Relaxation Rates for $S=1 / 2$ Molecular Species in Glassy Matrices or Magnetically Dilute Solids at Temperatures between 10 and 300 K. J. Magn. Reson. 1999, 139, 165-174.

(17) Escalera-Moreno, L.; Suaud, N.; Gaita-Ariño, A.; Coronado, E. Determining Key Local Vibrations in the Relaxation of Molecular Spin Qubits and Single-Molecule Magnets. $J$. Phys. Chem. Lett. 2017, 8 (7), 1695-1700. https://doi.org/10.1021/acs.jpclett.7b00479.

(18) Bader, K.; Winkler, M.; van Slageren, J. Tuning of Molecular Qubits: Very Long Coherence and Spin-Lattice Relaxation Times. Chem. Commun. 2016, 52 (18), $3623-$ 3626. https://doi.org/10.1039/C6CC00300A.

(19) Follmer, A. H.; Ribson, R. D.; Oyala, P. H.; Chen, G. Y.; Hadt, R. G. Understanding Covalent versus Spin-Orbit Coupling Contributions to Temperature-Dependent Electron Spin Relaxation in Cupric and Vanadyl Phthalocyanines. J. Phys. Chem. A 2020, 124 (44), 9252-9260. https://doi.org/10.1021/acs.jpca.0c07860.

(20) Albino, A.; Benci, S.; Tesi, L.; Atzori, M.; Torre, R.; Sanvito, S.; Sessoli, R.; Lunghi, A. First-Principles Investigation of Spin-Phonon Coupling in Vanadium-Based Molecular Spin Quantum Bits. Inorg. Chem. 2019, 58 (15), 10260-10268. https://doi.org/10.1021/acs.inorgchem.9b01407.

(21) Garlatti, E.; Tesi, L.; Lunghi, A.; Atzori, M.; Voneshen, D. J.; Santini, P.; Sanvito, S.; Guidi, T.; Sessoli, R.; Carretta, S. Unveiling Phonons in a Molecular Qubit with FourDimensional Inelastic Neutron Scattering and Density Functional Theory. Nat. Commun. 2020, 11 (1), 1751. https://doi.org/10.1038/s41467-020-15475-7. 
(22) Mirzoyan, R.; Hadt, R. G. The Dynamic Ligand Field of a Molecular Qubit: Decoherence through Spin-Phonon Coupling. Phys. Chem. Chem. Phys. 2020, 22 (20), 11249-11265. https://doi.org/10.1039/D0CP00852D.

(23) Lunghi, A. Ligand-Field Contributions to Spin-Phonon Coupling in a Family of Vanadium Molecular Qubits from Multi-Reference Electronic Structure Theory. ArXiv191204545 Cond-Mat Physicsquant-Ph 2019.

(24) Santanni, F.; Albino, A.; Atzori, M.; Ranieri, D.; Salvadori, E.; Chiesa, M.; Lunghi, A.; Bencini, A.; Sorace, L.; Totti, F.; Sessoli, R. Probing Vibrational Symmetry Effects and Nuclear Spin Economy Principles in Molecular Spin Qubits. Inorg. Chem. 2021, 60 (1), 140-151. https://doi.org/10.1021/acs.inorgchem.0c02573.

(25) Lunghi, A.; Totti, F.; Sessoli, R.; Sanvito, S. The Role of Anharmonic Phonons in UnderBarrier Spin Relaxation of Single Molecule Magnets. Nat. Commun. 2017, 8 (1), 14620. https://doi.org/10.1038/ncomms14620.

(26) Ballhausen, C. J. Introduction to Ligand Field Theory; McGraw-Hill, 1962.

(27) Solomon, E. I. Inorganic Spectroscopy - An Overview. Comments Inorg. Chem. 1984, 3, 227-320.

(28) Harris, D. C.; Bertolucci, M. D. Symmetry and Spectroscopy: An Introduction to Vibrational and Electronic Spectroscopy, New edition.; Dover Publications: New York, 1989.

(29) Jahn, H. A.; Teller, E.; Donnan, F. G. Stability of Polyatomic Molecules in Degenerate Electronic States - I-Orbital Degeneracy. Proc. R. Soc. Lond. Ser. - Math. Phys. Sci. 1937, 161 (905), 220-235. https://doi.org/10.1098/rspa.1937.0142.

(30) Atkins, P. W.; Friedman, R. S. Molecular Quantum Mechanics, 5th edition.; Oxford University Press: Oxford ; New York, 2010.

(31) Feynman, R. P. Forces in Molecules. Phys. Rev. 1939, 56 (4), 340-343. https://doi.org/10.1103/PhysRev.56.340.

(32) Wilson, R. B.; Solomon, E. I. Spectroscopic Studies of the Photoactive ${ }^{4} \mathrm{~T}_{2 \mathrm{~g}}$ Excited State of Hexaamminechromium(III). Inorg. Chem. 1978, 17 (7), 1729-1736. https://doi.org/10.1021/ic50185a006.

(33) Wilson, R. B.; Solomon, E. I. Spectroscopic Studies of Photochemically Important Transition Metal Excited States. 2. The ${ }^{1} \mathrm{~T}_{1 \mathrm{~g}},{ }^{3} \mathrm{~T}_{1 \mathrm{~g}}$, and ${ }^{5} \mathrm{~T}_{2 \mathrm{~g}}$ Excited States of Hexaamminecobalt(III). J. Am. Chem. Soc. 1980, 102 (12), 4085-4095. https://doi.org/10.1021/ja00532a018.

(34) Szilagyi, R. K.; Metz, M.; Solomon, E. I. Spectroscopic Calibration of Modern Density Functional Methods Using $\left[\mathrm{CuCl}_{4}\right]^{2-}$. J. Phys. Chem. A 2002, 106 (12), 2994-3007. https://doi.org/10.1021/jp014121c.

(35) Ariciu, A.-M.; Woen, D. H.; Huh, D. N.; Nodaraki, L. E.; Kostopoulos, A. K.; Goodwin, C. A. P.; Chilton, N. F.; McInnes, E. J. L.; Winpenny, R. E. P.; Evans, W. J.; Tuna, F. Engineering Electronic Structure to Prolong Relaxation Times in Molecular Qubits by Minimising Orbital Angular Momentum. Nat. Commun. 2019, 10 (1), 3330. https://doi.org/10.1038/s41467-019-11309-3.

(36) Fataftah, M. S.; Bayliss, S. L.; Laorenza, D. W.; Wang, X.; Phelan, B. T.; Wilson, C. B.; Mintun, P. J.; Kovos, B. D.; Wasielewski, M. R.; Han, S.; Sherwin, M. S.; Awschalom, D. D.; Freedman, D. E. Trigonal Bipyramidal $\mathrm{V}^{3+}$ Complex as an Optically Addressable Molecular Qubit Candidate. J. Am. Chem. Soc. 2020, 142 (48), 20400-20408. https://doi.org/10.1021/jacs.0c08986. 
(37) Maurice, R.; Bastardis, R.; Graaf, C. de; Suaud, N.; Mallah, T.; Guihéry, N. Universal Theoretical Approach to Extract Anisotropic Spin Hamiltonians. J. Chem. Theory Comput. 2009, 5 (11), 2977-2984. https://doi.org/10.1021/ct900326e.

(38) Legget, A. J.; Chakravarty, S.; Dorsey, A. T.; Fisher, M. P. A.; Garg, A.; Zwerger, W. Dynamics of the Dissipative Two-State System. Rev. Mod. Phys. 1987, 59 (1), 1-85.

(39) Schott, S.; McNellis, E. R.; Nielsen, C. B.; Chen, H.-Y.; Watanabe, S.; Tanaka, H.; McCulloch, I.; Takimiya, K.; Sinova, J.; Sirringhaus, H. Tuning the Effective Spin-Orbit Coupling in Molecular Semiconductors. Nat. Commun. 2017, 8 (1), 15200. https://doi.org/10.1038/ncomms15200.

(40) Camargo, L. C. de; Briganti, M.; Santana, F. S.; Stinghen, D.; Ribeiro, R. R.; Nunes, G. G.; Soares, J. F.; Salvadori, E.; Chiesa, M.; Benci, S.; Torre, R.; Sorace, L.; Totti, F.; Sessoli, R. Exploring the Organometallic Route to Molecular Spin Qubits: The [CpTi(Cot)] Case. Angew. Chem. 2021, 133 (5), 2620-2625. https://doi.org/10.1002/ange.202009634.

(41) Castle, J. G.; Feldman, D. W.; Klemens, P. G.; Weeks, R. A. Electron Spin-Lattice Relaxation at Defect Sites; E' Centers in Synthetic Quartz at 3 Kilo-Oersteds. Phys. Rev. 1963, 130 (2), 577-588. https://doi.org/10.1103/PhysRev.130.577.

(42) Castle, J. G.; Feldman, D. W. Resonance Modes at Defects in Crystalline Quartz. Phys. Rev. 1965, 137 (2A), A671-A673. https://doi.org/10.1103/PhysRev.137.A671. 